\title{
Caries en dentición primaria en infantes que acuden a la consulta de niño sano del ambulatorio docente del Hospital Universitario de Caracas
}

\author{
Saul Bermudez ${ }^{1}$ \\ María Elena Guerra ${ }^{2}$ \\ Humberto Gutierrez ${ }^{3}$ \\ Aura Y. Osorio ${ }^{4}$
}

\section{Resumen}

La caries en Dentición primaria en inglés conocida como Early Childhood Caries (ECC) ha sido definida por la Academia Americana de Odontología Pediátrica (AAPD) como la presencia de una o más lesiones cavitadas o de mancha blanca o superficie obturada de cualquier diente primario en niños entre 0 y 5 años. Objetivo: Determinar la presencia de caries en dentición primaria, en infantes, que acudieron la consulta de niños sano en Ambulatorio Docente del Hospital Universitario de Caracas, durante los meses de enero a junio durante el año 2014. Mé- todos: Se realizó un estudio prospectivo, transversal, descriptivo y se evaluó el índice ceo de 561 infantes que acudieron a solicitar algún tipo de tratamiento. Resultados: La prevalencia de caries fue de 80,6\%. El índice ceo promedio para el género femenino fue: ceo $=7.78$ y para el masculino: ceo $=7.58$. 65,95\% (370) de los niños presento algún tipo de tratamiento previo. El grupo etario más afectado correspondió al de 3 años. Conclusión: Los resultados indican una alta experiencia de caries en la población estudiada

Palabras clave: Caries dental; dentición primaria, niños.

Artigo Original

\section{Cárie dentária em dentição decídua em crianças que compareceram à consulta de crianças saudáveis no ambulatório docente do Hospital Universitario de Caracas}

\section{Resumo}

A cárie dentária em dentição decídua é conhecida em inglês como cárie de acomentimento precoce e é definida pela Academia Americana de Odontologia Pediátrica (AAPD) como a presença de uma ou mais lesões cavitadas ou de mancha branca ou superfície restaurada de

\footnotetext{
${ }^{1}$ Profesor Asistente. Especialista en Odontología Estética. Jefe del Departamento de Operatoria Dental y Estética

${ }^{2}$ Profesora Titular; Dra. En Ciencias Salud Bucal Materno Infantil, Coordinadora del Programa El Binomio Madre e Hijo VIH/SIDA del Centro de Atención a Pacientes con Enfermedades Infectocontagiosas "Dra. Elsa La Corte. Facultad de Odontología Universidad Central de Venezuela (CAPEI/ $U C V)$

${ }^{3}$ Profesor Titular. Médico Pediatra. Director del Ambulatorio Docente del Hospital Universitario de Caracas. Universidad Central de Venezuela

${ }^{4}$ Profesora Titular. Especialista en Periodoncia. Cátedra de Medicina Estomatológica. Facultad de Odontología Universidad Central de Venezuela Este estudio fue financiado por el Concejo Científico y Humanistico de la Universidad Central de Venezuela con el proyecto individual No.10-8616-20131
} 
qualquer dente decíduo em crianças entre 0 e 5 anos. Objetivo: Determinar a presença de cárie em dentição decídua, em crianças, que compareceram à consulta de crianças saudáveis no Ambulatório Docente do Hospital Universitário de Caracas, durante os meses de janeiro a junho de 2014. Métodos: Um estudo de seção transversal, descritivo foi realizado e o índice ceo de 561 das crianças que procuraram tratamento foi avaliado. Resultados: A prevalência de cárie dentária foi de $80,6 \%$. O ceo médio foi de 7,78 para o gênero feminino e 7,58 parao gênero masculino. 65,95\% (370) das crianças apresentaram algum tipo de tratamento prévio. O grupo etário mais afetado foi o de 3 anos. Conclusão: Os resultados indicam experiência elevada de cárie dentária na população estudada.

Palavras chaves: Cárie dentária; dentição decídua, crianças.

\section{Original article}

\section{Dental caries in the primary dentition, in children who attended the healthy children clinic of the University Hospital of Caracas}

\begin{abstract}
The American Academy of Pediatric Dentistry (AAPD) as the presence of one has defined caries in the primary dentition in English known as Early Childhood Caries (ECC) or more cavitated lesions or white spot or clogged surface of any primary tooth in children between 0 and 5 years. Objetive: To determine the presence of caries in the primary dentition, in children from 1 to 5 years of age, who attended for consultation at the healthy children mbulatory of the University Hospital of Caracas, during the months of January to June during the year 2014. Materials and methods: We conducted a prospective, cross-sectional, descriptive study and assessed index dmf of 561 children aged 1 to 5 than they went to request any type of treatment. Results: The prevalence of caries was $80.6 \%$. The index average $\mathrm{dmf}$ for women was: $\mathrm{dmf}=7.78$ and for men: ceo $=7.58$. 65,95 (370) $\%$ of children presented some sort of prior treatment. The most affected age group corresponded to 3 years old. Conclusion: The results indicate a high caries experience in the population studied.
\end{abstract}

Keywords. Dental Caries; primary dentition; children.

\section{Introducción}

La Organización Mundial de la Salud (OMS) ha definido la caries dental como un proceso localizado de origen multifactorial que se inicia después de la erupción dentaria, que es determinando por el reblandecimiento del tejido duro del diente y que evoluciona hasta formar una cavidad y si no se atiende oportunamente, afecta la salud general y la calidad de vida de los individuos de todas las edades ${ }^{1}$.

La presencia de caries dental en población en niños en período de dentición primaria, se asocia a una gran cantidad de variables, algunas de carácter biológico y otras que se relacionan con el medio ambiente, como hábitos higiénicos, dieta y consumo de sal fluorurada entre otros. La caries en dentición primaria constituye un problema de salud pública. La complejidad del proceso de caries dental en infantes involucra fac- 
tores socioeconómicos y culturales tales como pobreza, condiciones de vida precarias, bajo nivel educativo de los padres, estilos de vida y limitaciones en el acceso y disponibilidad de los servicios de salud bucal ${ }^{2: 3}$.

Se observa en la población estudiada un alto porcentaje de niños afectados por la caries dental, así como un índice ceo alto, al igual de lo que se esta reportando en el mundo, que a pesar de los esfuerzos y de los avances sobre los conocimientos sobre la caries, su prevención y control, se reporta que el problema no ha mejorado en las últimas décadas en los niños con dentición primaria ${ }^{4 ; 5}$.

Vargas y cols. En el 2006 realizaron un estudio en el cual afirman que por su carácter prevenible, la presencia de caries en niños en periodo de dentición primaria debería verse como una falla en el sistema de salud ${ }^{6}$ y que la salud bucal de los niños, en particular de aquellos en edad preescolar, es reflejo de inequidades en salud en la población, por cuanto éstos constituyen el grupo con mayores necesidades insatisfechas en salud bucal ${ }^{7}$.

Por lo anteriormente expuesto consideramos relevante examinar a niños que no van a buscar tratamiento odontológico, sino que van a la consulta del niño sano, bien sea a vacunarse o control, para enfatizar el conocimiento de la importancia de la caries dental ante los pediatras, por ser los infantes vulnerables en esta edad que dependen de los adultos, por lo que creemos que debemos tomar medidas especiales que corresponden a la odontopediatría, de manera que aseguren diagnóstico e intervención oportuna que cubra las necesidades de salud bucal de los infantes.

Este estudio se hizo con el objetivo de estimar la experiencia, frecuencia y niveles de severidad de caries dental en niños en periodo de dentición primaria, que asistieron a la Consulta de Niño Sano del Ambulatorio Docente del Hospital Universitario de Caracas.

\section{Material y métodos}

Se realizó un estudio observacional de tipo transversal sobre la frecuencia de caries dental en una muestra de 561 niños en dentición primaria, durante el periodo de enero a junio de 2014. La descripción metodológica sobre el tamaño y la selección de la muestra, así como los índices de caries obtenidos fue a convenir.

El protocolo de esta investigación fue aprobado por a comisión de bioética de la Facultad de Odontología, de la Universidad Central de Venezuela. Así con el consentimiento informado, Bajo el Número 0443-2013. De tal manera de informar al representante sobre el estudio y firmara sin presión, para en examen bucal, se tome en cuenta el asentamiento del niño, de manera que permitiera participar de manera voluntaria.

Se utilizó el instrumento de la cátedra de Preventiva y Social de la Facultad de Odontología para determinar el índice ceo, por ser el índice más utilizados en la valoración de la caries para dientes primarios son: ceo que es el promedio de dientes afectados por individuo.

El examen clínico y registro de caries, clínico, siguiendo los criterios de la OMS8:9. Se empleo El índice ceo, fue propuesto por Gruebbel en $1944^{10}$ y cuenta con las siguientes características: Se lo utiliza en dentición primaria, escribiéndose en letras minúsculas y se ignoran los dientes faltantes porque la mayoría se exfolian en forma natural. Se utilizó también el índice de Knutson para clasificar a los grupos de niños en porcentajes en cuanto a presencia y ausencia de la misma ${ }^{11}$.

El examen bucal lo realizó una odontopediatra calibrada para tal fin, ella obtuvo un Kappa intra-examinador de 0,77 (IC 0,70-0,83). El examen se realizo en posición rodilla con rodilla entre el representante y la examinadora, en la sala de es- 


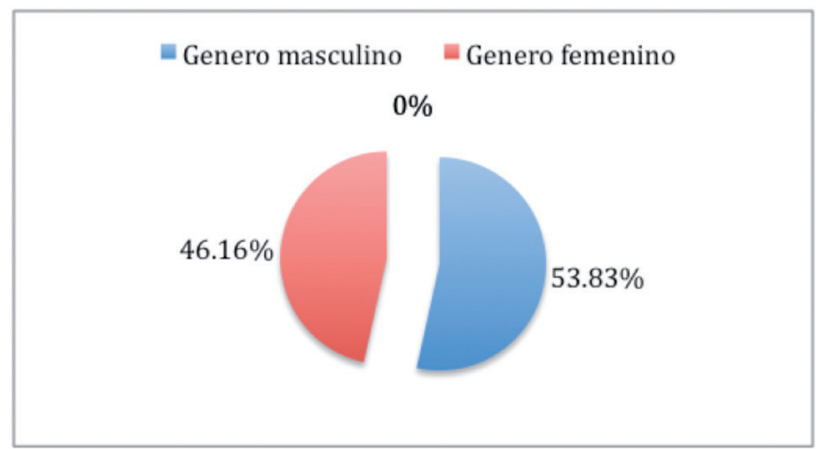

Figura 1. Distribución de acuerdo al género de los participantes del estudio. Consulta de Niño Sano. Ambulatorio Docente del Hospital Universitario 2014.

pera, con luz natural, ayudada por baja lengua cumpliendo las medidas de bioseguridad.

Los datos fueron transcritos y analizados con el programa computarizado SPSS y conformados en figuras y tablas.

\section{Resultados}

Los niños estudiados estuvieron en edades comprendidas ente 1 a 5 años, con una edad promedio del grupo de estudio de 3,6 $\pm 1,1$ años.

La población total examinada fue de 561 infantes estando distribuida en $46.16 \%$ (259) por

Tabla 1. Distribución según el índice ceo de acuerdo al género. Consulta de Niño Sano. Ambulatorio Docente Hospital Universitario 2014.

\begin{tabular}{|c|c|c|}
\hline GÉNERO & $\begin{array}{c}\text { Número de } \\
\text { pacientes }\end{array}$ & INDICE CEO \\
\hline Femenino & 259 & 7.78 \\
\hline Masculino & 302 & 7.50 \\
\hline TOTAL & 561 & 7.69 \\
\hline
\end{tabular}

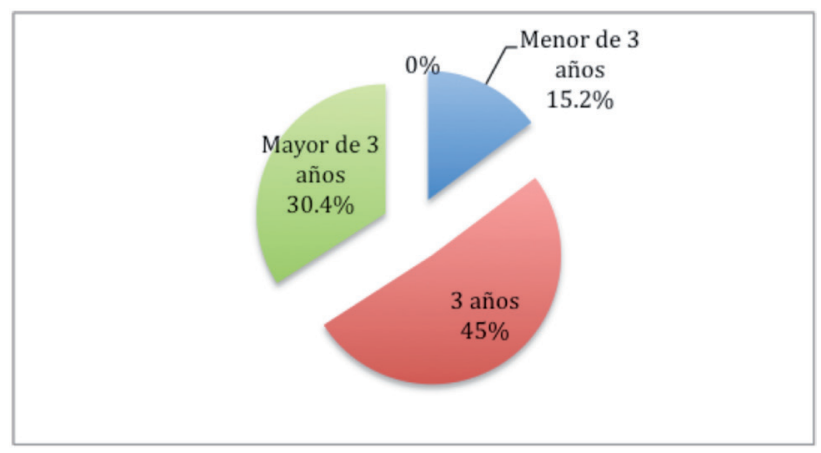

Figura 2. Distribución de acuerdo al porcentaje de caries en los niños estudiados. Consulta de Niño Sano. Ambulatorio Docente Hospital Universitario 2014.

el grupo femenino y 53.83\% (302) por el grupo masculino (Figura 1).

En relación a la frecuencia de caries se observó que un $80.6 \%$ (448) de la población total del estudio siendo el grupo etario más afectado el de los 3 años, con un $45 \%$ (252); seguido por los niños mayores de 3 años 30.4\% (168) y 15.2\% (84) en los menores de 3 años (Figura 2).

Los índices de caries en la población estudiada fueron observados de la siguiente manera, para el género femenino ceo $=7.78$ y para el género masculino: ceo $=7.50$ (Tabla 1$)$.

Tabla 2: Distribución según el porcentaje de niños del estudio que recibieron algún tipo tratamiento odontológico. Consulta de Niño Sano. Ambulatorio Docente Hospital Universitario 2014.

\begin{tabular}{|c|c|c|}
\hline $\begin{array}{c}\text { Tratamiento } \\
\text { Odontológico }\end{array}$ & $\begin{array}{c}\text { Número de } \\
\text { pacientes }\end{array}$ & Porcentaje \\
\hline Extracciones & 155 & $81.15 \%$ \\
\hline $\begin{array}{c}\text { Recidiva en } \\
\text { restauraciones }\end{array}$ & 36 & $18.84 \%$ \\
\hline TOTAL & 191 & $100 \%$ \\
\hline
\end{tabular}


De la población total no habían recibido ningún tipo de tratamiento, 65,95\% (370) y si habían recibido algún tipo de atención odontológica en sus dientes primarios un 34.5\% (191). Debemos destacar que un $81.15 \%$ (155) habían perdido al menos un diente primario por causa de caries, $18.84 \%$ (36) presentaban restauraciones con recidiva de caries en dientes primarios (Tabla 2).

\section{Discusión}

La alta prevalencia de caries observada en $80.6 \%$ de la población, muestra en el género femenino presentó un índice ceo $=7.78$ y para el masculino el índice ceo $=7.50$ afectados por individuos considerando la suma de dientes primarios. Es posible que las diferencias en los valores de los índices de caries más alto entre el género femenino con relación al masculino, se expliquen de manera parcial ante el hecho de que en el límite de edad de los grupos de estudio se presenta en las niñas una cronología de erupción dental más temprano que los niños, dando como consecuencia que los dientes en las niñas tengan mayor tiempo de exposición al riesgo de caries. Según la literatura la caries afecta más a la población femenina; ${ }^{14-16}$ Raitio y col. ${ }^{17}$ mencionan que los indicadores de riesgo para caries dental no son iguales para los niños que para las niñas; sin embargo, el factor edad contribuye sólo de manera parcial a la explicación en el comportamiento de la caries. Los resultados conducen a pensar en la necesidad de identificar los factores que están impactando en los perfiles de caries encontrados en la población de estudio.

A diferencia de otros estudios ${ }^{18}$ que refieren haber observado que la prevalencia de caries en dentición primaria corresponde a caries no cavitadas mientras que el nuestro las lesiones que describimos y observamos fueron cavitadas.
En nuestro estudio que se observó un mayor porcentaje de caries a los 3 años, lo que difiere con otro estudio realizado en Taiwan ${ }^{19}$ en el cual los autores refieren que el porcentaje superior de caries se observo a los 5 años, ya que incrementa el riesgo por el aumento de consumos de dulces, inicia etapa de erupción de dentición permanente que pudiera ocasionar incomodidades al cepillado dental.

Se observó que de la población total (561), 34.5\% (191) habían recibido algún tipo de tratamiento odontológico, también es de destacar que a mayor número de dientes afectados, menor número de niños atendidos; pareciera que existe dificultades para atender oportunamente la salud bucal de estos pequeños. Otro punto que llama la atención, se relaciona con el numero de niños que mostraron recidiva en dientes que habían sido restaurados $18.84 \%$ (36) en dientes primarios, con respecto a la población total, lo que habla de la susceptibilidad a la patología más alta a lo reportado por Chestnut IG y $\mathrm{col}^{20}$, en adolescentes escoceses quienes han reportado que bajo los mejores cuidados de atención, prevención y educación en salud, se observa $10 \%$ de recidiva de caries.

Del 34.5\% (191) de los niños que habían recibido atención en dientes primarios, $81.15 \%$ (155) habían perdido al menos un diente primario por causa de la caries avanzadas, se pudiera pensar que para un número importante de niños la única atención dental recibida pudo haber sido la extracción, se resalta que la atención dental predominante fue la extracción. Se encontraron estudios realizados en Venezuela que reportan perdida prematura de dientes primarios que traen como consecuencia maloclusiones dentarias ${ }^{21}$.

A pesar que el flúor es el elemento más efectivo contra la caries dental ${ }^{13}$. Llama la atención que los representantes de los niños del estudio no re- 
firieron uso de aplicaciones de flúor como agente preventivo a diferencias de otras investigaciones como en Perinetti ${ }^{22}$ en un estudio realizado en 13 provincias de Italia encontró en niños entre 2 y 5 años que el uso regular de suplementos de flúor resultaron protectores para desarrollar caries dental.

Con el criterio actual sobre la flora ecológica de la caries dental, se sabe que proviene de los cuidadores con lesiones cariosas activas, que puede transmitir la patología al infante con dientes recién erupcionados, además sin control adecuado de dieta no cariogenica ${ }^{2 ; 5}$. Se destaca que los representantes de esta población no sabian que la caries dental es de etiología multifactorial, transmisible, que es posible su prevención por medio de educación sobre los correctos hábitos de higene bucal, cepillado dental al erupcionar el primer diente, disminuir ingesta de alimentos azucarados, evitar en los lactantes el tiempo prologado de biberones así como también soplar los alimentos para enfriarlos y sobre todo la importancia de la colocación de barnices de fluoruro por el odontopeditra para su efectiva prevención ${ }^{2 ; 5 ; 23 ; 24}$.

\section{Conclusión}

Los resultados indican una alta experiencia de caries en la población estudiada, por lo que se destaca que la caries en dentición primaria compromete la calidad de vida, de la población motivo de estudio.

\section{Recomendación}

Se hace imperativo que las políticas públicas se organicen acciones dirigidas a los niños menores de seis años, dentro de un enfoque integral de salud que promueva su crecimiento y desarrollo, en coherencia con acciones de promoción de la salud en búsqueda de mejores condiciones de vida, sin descuidar acciones de prevención de la caries, mediante estrategias que involucren a los representantes en los avances en la comprensión de la enfermedad más común en humanos.

\section{Referencias}

1. World Health Organization: Oral health surveys. Basic Methods. 1987. 3rd. Geneve, Suiza, WHO.

2. Ismail AI, Sohn W. The impact of universal access to dental care on disparities in caries experience in children. J Am Dent Assoc 2001; 132 (3): 295-303

3. Selwitz RH, Ismail AI, Pitts NB. Dental caries. Lancet 2007; 369 (9555): 51-59

4. Pitts N, Harker R. Children's dental health in the United Kingdom 2003: obvious decay experience. London: Officeof National Statistics; 2005

5. Beltrán-Aguilar ED, Barker LK, Canto MT, Dye BA, Gooch BF, Griffin SO, Hyman J, Jaramillo F, Kingman A, NowjackRaymer R, Selwitz RH, Wu T; Centers for Disease Control and Prevention (CDC).MMWR Surveill Summ. 2005 Aug 26;54(3):1-43.

6. Vargas CM, Ronzio CR. Disparities in early childhood caries. BMC Oral Health 2006; 6 Supl 1: S3.

7. Crall JJ. Access to oral health care: professional and societal considerations. J Dent Educ 2006; 70: 1133-1138

8. World Health Organization. Oral Health Surveys, sic methods. Fourth ed. World Health Organization, Geneva, 1997:40-47

9. Who Oral Health Country. Caries Prevalence; DMFT and DMFTS. www.whocollab.od.mah.se

10.Protocolo indicadores epidemilógico.Comisión Nacional de Prevención Nacional CORA http:/ / usuarios.advance.com. ar 
11. Klein, H.; Palmer, C. E. \& Knutson, J. W. Studies on Dental Caries, I, Dental status and Dental Needs of elementary school. Pub. Health Rep., 1938. 53:751-65.

12. Milen A, Hausen H, Heinonen O, Paunio I. Caries in primary dentition related to age, sex, social status, and county of residence in Finland. Dent Oral Epidemiol. 1981; 9:83-6.

13. Evans W, Donald J, Brown H, Silva P. Relationship between fluoridation and socioeconomic status on dental caries experience in 5-year-old New Zealand children. Community Dent Oral Epidemiol. 1984; 12:5-9.

14. Evans R, Edward CM, Brian W, Darvell A. Determinants of variation in dental caries experience in primary teeth of Hong Kong children aged 6-8 years. Community Dent Oral Epidemiol. 1993; 21:1-3.

15. Wei SH, Holm AK, Tong LS, Yuen SW. Dental caries prevalence and related factors in 5 year old children in Hong Kong. Pediatr Dent. 1993; 15: 116-9.

16. Kerosuo H, Honkala E. Caries experience in the primary dentition of Tanzanian and Finnish 3-7 year old children. Community Dent Oral Epidemiol. 1991; 19:272-6.

17. Raitio M, Pienihakkinen K, Scheinin A. Multifactorial modeling for prediction of caries increment in adolescents. Acta Odontol Scand. 1996; 54: 118-21.

18. Autio-Gold JT; Tomar SL. Prevalence of noncavitated and cavitated carious lesions in 5 years old head start schoul clidren in Alachua County, Florida. Pediatr Dent 2005; 27:54-60

19. Tsai A.ChenCh: Li 1; Hsiang Ch; Hsu K. Risk indicators for early chilhood caries in Taiwan Community Oral Dent Epidemiology 2006; 34; 437-445

20. Chestnut IG, Jones PR, Jacobson AP, Schafer F, Stephen KW. Prevalence of clinically apparent recurrent caries in Scottish adolescents and the influence of oral hygiene practice. Caries Res. 1995; 29: 266-71

21. Hernández, Johana, Montiel, Liliana, Velásquez, Josmary Carolina Alcedo. Djurisic, Aura. Oscar Quirós Álvarez, Lennys Molero, Jorge Tedaldi Influencia de la perdida prematura de dientes primarios por caries dental, como causa de maloclusiones en pacientes de 7 a 10 años que acuden al Servcio de Odontología del Centro de Atención Interal de Salud Francisco de Miranda. http: / / www.ortodoncia.ws / publicaciones / 2010

22. PerineteG: Caputi S:VarvaraG. Risk/ prevention Indicators for the prevalence of dental caries in Schoolchildren:Resoults from the Italiam OHSRA Survey. Aries research 2005;39:92-99

23. Bowen WH, Lawrence RA. Comparison of the cariogenicity of cola, honey, cow milk, human milk, and sucrose. Pediatrics. 2005 Oct;116(4):921-6.

24. Guerra María E; Hernández Maria N; Carrasco W; Albornoz E. Intervención Odontosanitaria para controlar caries en dentición primaria en hijos de madres VIH/SIDA Acta Odontológica Venezolana 2014 Vol. 52; No 1.

Recibido: 03-08-14

Aceptado: 12-09-14

Correspondencia: bermudes66.s@gmail.com 